\title{
Myopathy due to primary systemic amyloidosis
}
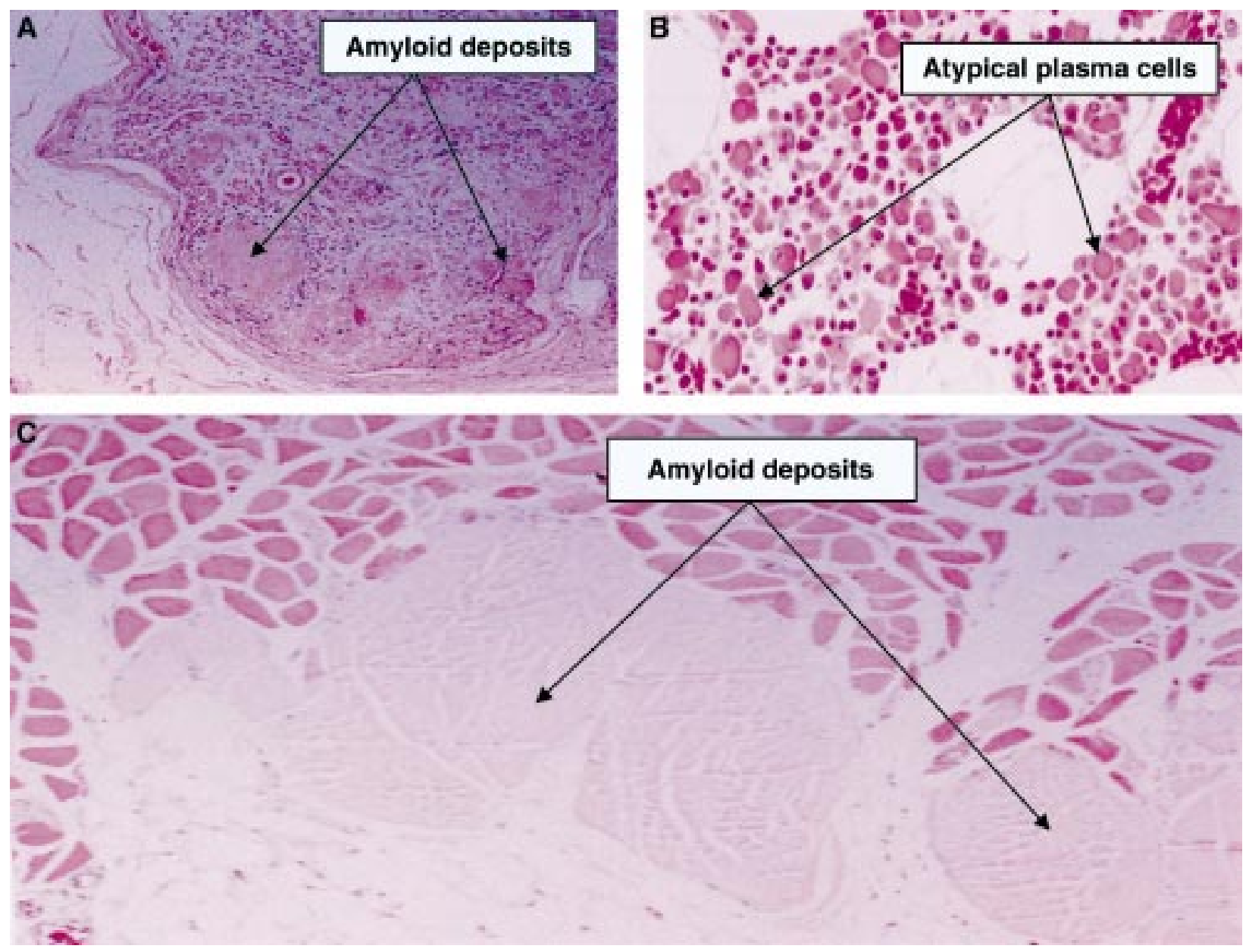

(A) Section of sciatic nerve demonstrating amyloid deposition within the nerve bundles. (B) Bone marrow showing a diffuse infiltrate of atypical secretory plasma cells. (C) Deltoid muscle showing extensive interstitial amyloid deposition.

An 85 year old man presented with a 6 month history of progressive proximal muscle weakness, with associated paraesthesia, and dysphagia. Clinical examination showed signs of a peripheral neuropathy, proximal muscle wasting, and weakness of the lower limbs. Electrophysiological examination demonstrated a generalised axonal sensori-motor neuropathy, with a superimposed proximal myopathy. Creatinine kinase concentration and erythrocyte sedimentation rate were normal, as were blood lactate and carotene concentrations. A deltoid muscle biopsy showed ragged red fibres, with no features of an inflammatory process. The patients' condition deteriorated rapidly, culminating in a cardiac respiratory arrest.

On postmortem examination, there was extensive deposition of amyloid multiple organs including the heart, lungs, oesophagus, liver, spleen, kidney, prostate, and thyroid. Sections of nerves from the brachial plexus, and sural and sciatic nerves showed amyloid deposition in the vessel walls, as well as focal deposition within nerve bundles (figure A). The bone marrow appearance was consistent with multiple myeloma, with a diffuse infiltrate of atypical plasma cells containing abundant eosinophilic cytoplasmic inclusions (figure B). The plasma cells stained strongly for kappa light chains and negatively for lambda light chains, indicating monoclonality. The deltoid and quadriceps muscles showed coagulative necrosis with amyloid deposits within the vessel walls and the interstitium between muscle fibres (figure $\mathrm{C}$ ).

Amyloid myopathy should be considered when a patient presents with progressive proximal muscle weakness, ${ }^{12}$ even in the absence of accompanying pseudohypertrophy of muscles, hoarseness of voice, macroglossia, or palpable nodules within muscles. ${ }^{3}$ Deposition of amyloid is often patchy and may not be seen on a limited muscle biopsy. ${ }^{4}$

ARUN AGGARWAL ALASTAIR CORBETT

Department of Neurology, Concord Repatriation General Hospital,

Hospital Road, Concord, NSW, Australia 2139

1 Nadkarni N, Freimer M, Mendell JR. Amyloidosis causing a progressive myopathy. Muscle Nerve 1995;18:1016-18.

2 Jennekens FGI, Wokke JHJ. Proximal weakness of the extremities as the main feature of amyloid myopathy. F Neurol Neurosurg Psychiatry 1987;50: $1353-8$

3 Ringel SP, Claman HN. Amyloid-associated muscle pseudohypertrophy. Arch Neurology 1982;39:413-17.

4 Gertz MA, Kyle RA. Myopathy in primary systemic amyloidosis. 7 Neurol Neurosurg Psychiatry 1996;60:655-60. 